\title{
Análise de grafos de visibilidade do mercado brasileiro de soja, milho e carne de
}

\author{
frango \\ Analysis of the visibility graphs of the Brazilian soybean, corn and chicken meat market \\ Análisis de grafos de visibilidad del mercado brasileño de soja, maíz y carne de pollo
}

José Edvaldo de Oliveira Nunes ORCID: https://orcid.org/0000-0003-4417-1875

Universidade Federal Rural de Pernambuco, Brasil

E-mail: edvaldooliveiranunes@ outlook.com

Joelma Mayara da Silva

ORCID: https://orcid.org/0000-0003-2354-6068

Universidade Federal Rural de Pernambuco, Brasil

E-mail: jms.estat@gmail.com

Lidiane da Silva Araújo

ORCID: https://orcid.org/0000-0003-3111-7925

Universidade Federal Rural de Pernambuco, Brasil

E-mail: lidiane_sac@hotmail.com

Guilherme Rocha Moreira

ORCID: https://orcid.org/0000-0001-6344-1151

Universidade Federal Rural de Pernambuco, Brasil

E-mail: guirocham@gmail.com

Tatijana Stosic

ORCID: https://orcid.org/0000-0002-5691-945X

Universidade Federal Rural de Pernambuco, Brasil E-mail: tastosic@gmail.com

Borko Stosic

ORCID: https://orcid.org/0000-0001-5031-6968

Universidade Federal Rural de Pernambuco, Brasil

E-mail: borkostosic@gmail.com

\begin{abstract}
Resumo
A produção, exportação e consumo doméstico de carne de frango no Brasil crescem constantemente, apresentando para esse produto variações de preço que comprometem o orçamento da população. O preço dessa commodity depende, principalmente, do custo da ração, que inclui o milho e a soja como fonte de energia e proteína, respectivamente. Estudar a dinâmica de preço desse produto requer, portanto, uma investigação também acerca dos preços dos insumos milho e soja. Com o objetivo de contribuir para o desenvolvimento e validação de modelos teóricos e computacionais para previsão dos preços de soja, milho e carne de frango, analisaram-se aqui seus preços diários (em moeda brasileira), registrados entre 03/01/2011 e 04/12/2019, obtidos do CEPEA/ESALQ/USP. A partir das séries temporais dos dados de preços originais, foram também criadas as séries de retorno e de volatilidade. Foi utilizado o método Grafo de Visibilidade Horizontal (HVG), implementado em linguagem C, para mapear as séries temporais em redes complexas e, então, calcular os índices topológicos de interesse. Os resultados mostraram que as redes dos preços da soja e do milho são menos integradas que a da carne de frango. Além disso, as séries originais dos preços da soja e do milho, bem como a série de retorno dos preços do frango e a de volatilidade dos preços de milho, são geradas por processos estocásticos correlacionados; a série de retorno dos preços de soja apresenta comportamento com tendência ao de processos não correlacionados; e as demais séries são governadas por processos caóticos.
\end{abstract}

Palavras-chave: Mercado agrícola; Redes complexas; Grafo de visibilidade horizontal.

\begin{abstract}
The production, export and consumption of chicken meat in Brazil are constantly growing, presenting price variations for this product that compromise the population's budget. The price of this commodity depends mainly on the cost of the feed, which includes corn and soy as a source of energy and protein, respectively. The study of the price dynamics of this product, therefore, requires an investigation also on the prices of corn and soybean inputs. In order to contribute to the development and validation of theoretical and computational models for the projection of prices for soybeans, corn and chicken, were analyzed their daily prices (in BRL), registered between 03/01/2011 and 12/04/2019, obtained of CEPEA/ESALQ/USP. From the time series of the original data of prices, were also created the series of return and volatility. The Horizontal Visibility Graph (HVG) method, implemented in C language, was
\end{abstract}


used to map the time series in complex networks and, thereafter, calculate the topological indexes of interest. The results showed that the soybean and corn price networks are less integrated than that of chicken meat. In addition, the original series of soybean and corn prices, as well as the series of return of chicken prices and the volatility of corn prices, are generated by correlated stochastic processes; the return series of soybean prices presents behaviour that tends to the one of uncorrelated processes; and the others series are generated by chaotic processes.

Keywords: Agricultural market; Complex networks; Horizontal visibility graph.

\section{Resumen}

La producción, exportación y consumo interno de carne de pollo en Brasil está en constante crecimiento, presentando variaciones de precio de este producto que comprometen el presupuesto de la población. El precio de esta commodity depende principalmente del costo del alimento, el cual incluye maíz y soja como fuente de energía y proteína, respectivamente. Estudiar la dinámica de precios de este producto requiere, por tanto, una investigación también sobre los precios de los insumos maíz y soja. Con el fin de contribuir al desarrollo y validación de modelos teóricos y computacionales para pronosticar precios de soja, maíz y carne de pollo, se analizaron aquí sus precios diarios (en moneda brasileña), registrados entre 01/03/2011 y 04/12/2019, obtenidos de CEPEA/ESALQ/USP. A partir de las series temporales de precios originales, se crearon también las series de retorno y de volatilidad. Se utilizó el método Grafo de Visibilidad Horizontal (HVG), implementado en lenguaje C, para mapear las series temporales en redes complejas y luego calcular los índices topológicos de interés. Los resultados mostraron que las redes de precios de soja y de maíz están menos integradas que la de carne de pollo. Además, la serie original de precios de la soja y el maíz, así como la serie de retorno de los precios del pollo y la serie de volatilidad del maíz, se generan mediante procesos estocásticos correlacionados; la serie de retorno de precios de soja presenta comportamiento que tiende al de procesos no correlacionados; y las otras series son generadas por procesos caóticos.

Palabras clave: Mercado agrícola; Redes complejas; Grafo de visibilidad horizontal.

\section{Introdução}

De acordo com os dados fornecidos pelo Foreign Agricultural Service / United States Department of Agriculture (FAS/USDA, 2020) sobre exportação e consumo interno de produtos primários brasileiros, pode-se concluir que esses produtos exercem papel fundamental na economia do Brasil devido, principalmente, ao seu relevante percentual de participação nas exportações mundiais, como também ao alto consumo interno. Entre suas principais commodities, a carne de frango é a que merece maior destaque. Isso porque o País ocupa a segunda posição no ranking mundial de produção, com cerca de 12,86 milhões de toneladas, e o primeiro lugar como exportador, com mais 4 milhões de toneladas (CNA, 2020). Segundo a EMBRAPA (2020), 68\% da produção de carne de frango em 2019 foi destinada ao mercado interno. O aumento do consumo dessa commodity causa alterações em seus preços, trazendo impacto ao orçamento dos brasileiros. O milho como fonte de energia e a soja como fonte de proteína (Gouveia, de Paulo, da Silva, de Sousa, dos Santos, \& Minafra, 2020) são os principais insumos utilizados na formulação das rações de frangos de corte (Oliveira Júnior, Wander, \& Figueiredo, 2014).

O valor bruto da produção (VBP) agropecuária brasileira alcançou 651,5 bilhões de reais em 2019, dos quais 400,7 bilhões na produção agrícola e 250,8 bilhões no segmento pecuário. A soja em grãos é a principal responsável pelo faturamento brasileiro no setor agropecuário e o milho é o responsável pelo terceiro maior VBP brasileiro (CNA, 2020).

O mercado de commodities agrícolas tem atraído considerável atenção de pesquisadores de diversas áreas devido ao aumento dos preços desses produtos no início do ano de 2001. A maior elevação de preço se deu com a crise alimentar que ocorreu em 2007/2008, e posterior aumento em 2010/2011 (Hochman, Rajagopal, Timilsina, \& Zilberman, 2014), provocando insegurança alimentar, especialmente nos países em desenvolvimento (Ivanic, Martin, \& Zaman, 2012). Diversas técnicas têm sido empregadas para estudar a dinâmica de preços das commodities soja, milho e carne de frango, em particular. Oliveira Júnior et al. (2014) estudaram as relações lineares entre os preços de atacado de milho, soja e frango in natura, utilizando técnicas de análises estatísticas de correlação e regressão lineares, e observaram a existência de forte correlação linear entre os preços da carne de frango e milho, com o coeficiente de Pearson de 0,648, e carne de frango e soja, com 0,718. Bini et al. (2016) também investigaram a relação entre os preços dessas três commodities brasileiras, com a abordagem econométrica da Análise de Autorregressão Vetorial (VAR), e observaram que: variações no preço da soja são transmitidas para o preço do 
milho; os preços do milho e do frango apresentam dependência mútua entre si; e os preços da soja não sofrem influência dos demais produtos. Santana et al. (2020) estudaram a dinâmica dos preços brasileiros do milho, da soja e da carne de frango, utilizando a técnica de Análise de Quantificação de Recorrência. Seus resultados mostraram que os preços da soja e do milho são mais sincronizados entre si do que entre algum deles e os da carne de frango. Além disso, revelaram que a variação temporal dos preços da carne de frango recebe maior influência do valor da soja que do de milho.

Diante do exposto, nota-se que cada técnica já empregada para investigar a dinâmica de preços das três commodities agrícolas aqui analisadas, forneceu informações novas e úteis do ponto de vista tanto científico quanto econômico, e que compreender as interações e impactos desse mercado agrícola é importante para se estabelecer uma base científica. Visando contribuir para a base de desenvolvimento e validação dos modelos teóricos e computacionais para previsão de preços de soja, milho e carne de frango, utilizou-se neste trabalho o método Grafo de Visibilidade Horizontal (Horizontal Visibility Graph HVG), desenvolvido por Luque et al. (2009) para análise de séries temporais com base na teoria de redes complexas. Foram então analisadas as séries originais, bem como as de retorno e volatilidade dos preços diários de soja, milho e carne de frango, com o objetivo de investigar o tipo de sistema complexo pelos quais essas séries são geradas e, assim, obter informações úteis sobre a dinâmica de preços dessas commodities.

\section{Referencial Teórico}

$\mathrm{Na}$ literatura atenção especial se tem dado ao uso da teoria de redes complexas para a análise de séries temporais não lineares (Zhang, Sun, Luo, Zhang, Nakamura, \& Small, 2008). A descoberta de comportamentos complexos de séries temporais não lineares constitui um problema fundamental de interesse contínuo, devido a sua importância em diversas áreas de pesquisa (Gao, Cai, Yang, Dang, \& Zhang, 2016) que buscam, cada vez mais, compreender as interações complexas que resultam em processos estocásticos ou caóticos com o auxílio de métodos capazes de distinguir um do outro (Luque, Lacasa, Ballesteros, \& Luque, 2009). Assim a combinação da abordagem de análise de séries temporais não lineares com a teoria de redes complexas, já sendo amplamente aceita no campo das ciências de sistemas complexos com fortes ligações com a Dinâmica não Linear e a Física Estatística, tornou-se um campo de estudo importante com variedade de aplicações em vasta gama de disciplinas (Zou, Donner, Marwan, Donges, \& Kurths, 2019).

Historicamente o estudo sobre redes complexas tem foco na teoria dos grafos, em que um grafo é definido como um conjunto de nós (vértices) e ligações (arestas) entre eles (Boccaletti, Latora, Moreno, Chavez, \& Hwang, 2006). Assim, para tornar as séries temporais acessíveis a técnicas complexas, é necessário transformá-las em uma representação de rede por meio de um algoritmo que defina vértices e arestas. Há pelo menos três maneiras de se transformarem séries temporais em redes complexas: (i) similaridade estatística mútua, que gera redes de proximidades; (ii) convexidade de observações sucessivas, com que se obtêm grafos de visibilidade; e (iii) probabilidade de transição, que gera redes de transição (Zou et al., 2019). Entre esses métodos, um que se tem destacado é o Grafo de Visibilidade (Visibility Graph - VG), que considera um critério de "visibilidade" para determinar a ligação entre dois nós da rede gerada, associados a pontos de dados da série temporal em análise. Esse método possui duas formas, o Grafo de Visibilidade Natural (VG) (Lacasa, Luque, Ballesteros, Luque, \& Nuno, 2008) e o Grafo de Visibilidade Horizontal (HVG) (Luque et al., 2009) - abordagem utilizada neste trabalho. O HVG tem sido utilizado para a análise de dados em Fisiologia (Zhu, Li, \& Wen, 2014; Madl, 2016; Ahmadi \& Pechenizkiy, 2016), Hidrologia (Braga, Alves, Costa, Ribeiro, De Jesus, Tateishi, \& Ribeiro, 2016; Lange, Sippel, \& Rosso, 2018) e Finanças (Vamvakaris, Pantelous, \& Zuev, 2018).

\subsection{Grafo de Visibilidade Horizontal}

Subgrafo do Grafo de Visibilidade Natural, o Grafo de Visibilidade Horizontal (HVG) considera, na sua formação, 
certo critério geométrico de visibilidade. Ele é assim obtido: seja $\left\{x_{i}\right\}_{i=1, \ldots, N}$ uma série temporal de $N$ dados. $\mathrm{O}$ algoritmo associa cada dado da série temporal a um nó (ou vértice) no grafo de visibilidade horizontal a ser construído. Dois nós $i$ e $j$ do grafo são conectados entre si somente se for possível traçar uma linha horizontal na série temporal unindo a linha vertical correspondente ao dado $x_{i}$ à do dado $x_{j}$ e que não intercepte nenhuma outra linha vertical intermediária de um dado da série. Logo, dois nós serão conectados de acordo com o seguinte critério geométrico de visibilidade horizontal:

$$
x_{i}, x_{j}>x_{n} \text { para todos os } n \text { tais que } i<n<j .
$$

Um exemplo ilustrativo do algoritmo HVG é apresentado na Figura 1. Cada dado na série (Figura 1(a)) corresponde a um nó no grafo (Figura 1(b)), enumerado segundo a correspondência temporal do respectivo dado da série. Dois nós do grafo são conectados se suas alturas de dados correspondentes forem maiores do que todas as alturas de dados entre eles. As linhas horizontais na série indicam as possíveis conexões do grafo, estabelecidas de acordo com o critério geométrico. A altura de cada barra vertical indica um dado da série temporal. Deve-se notar que, por exemplo, foi possível traçar linhas entre o dado $x_{5}$ e os dados $x_{3}, x_{4}$ e $x_{6}$ sem que nenhuma barra vertical fosse interceptada. Qualquer outra conexão entre esse dado $\left(x_{5}\right)$ e os demais violaria o critério de visibilidade descrito em (1). Assim, o nó $i=5$ do grafo de visibilidade gerado está conectado apenas aos nós $j=3, j=4$ e $j=6$.

Figura 1. Exemplo ilustrativo de uma série temporal representada por barras verticais (a) e seu correspondente grafo de visibilidade gerado pelo algoritmo HVG (b).

(a)

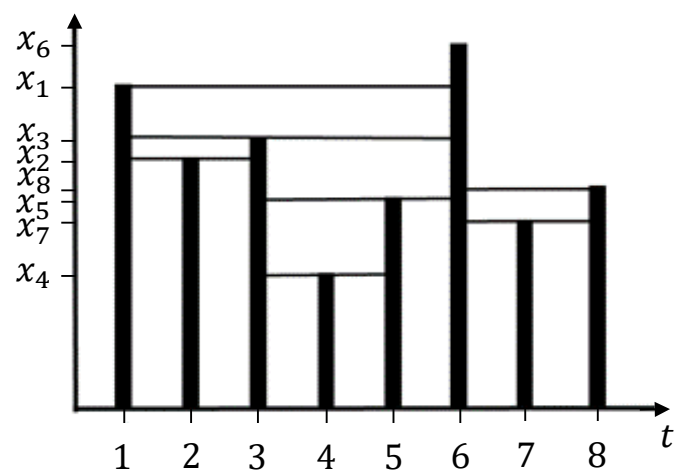

(b)

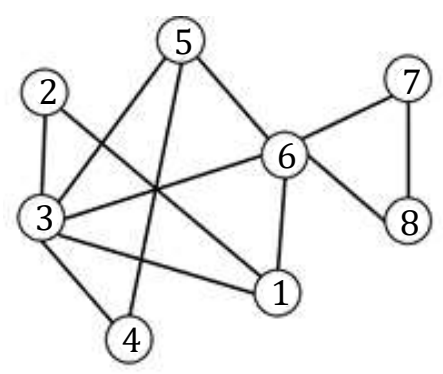

Fonte: Luque et al. (2009). Adaptado pelos autores.

Os nós de uma rede complexa podem apresentar diferentes quantidades de conexões, como se pode observar no grafo apresentado na Figura 1(b). Uma rede obtida do algoritmo VG ou HVG pode, ainda, ser representada por sua matriz de adjacência $A=\left[a_{i j}\right]$, em que $a_{i j}=1$ se os nós $i$ e $j$ se encontram conectados, e $a_{i j}=0$, caso contrário. A matriz $A$ pode ser usada para calcular o número de conexões $k$ de um nó, chamado de grau do nó, quantidade com a qual se podem extrair diversas medidas para a topologia da rede (Sivakumar \& Woldemeskel, 2015), tais como: o Coeficiente $\lambda$ da distribuição de grau do nó, o Coeficiente de Agrupamento e o Comprimento Médio do Menor Caminho, que são os índices calculados neste trabalho. A análise dos índices topológicos das redes complexas é o que permite classificar os sistemas dinâmicos que governam as séries temporais em estudo. 


\section{2 Índices topológicos do Grafo de Visibilidade Horizontal}

\subsubsection{Coeficiente da distribuição do grau de nó}

A distribuição de graus dos nós é definida por uma função de distribuição $P(k)$ e fornece a probabilidade de um nó selecionado aleatoriamente ter exatamente $k$ conexões. Em um grafo puramente aleatório, por exemplo, uma vez que as conexões são colocadas aleatoriamente, a maioria dos nós tem aproximadamente o mesmo grau, o que se aproxima do grau médio $\bar{k}$ da rede (Albert \& Barabási, 2002).

Lacasa e Toral (2010) mostraram que uma série mapeada em um grafo de visibilidade horizontal exibe distribuição exponencial de graus dos nós na forma $P(k) \sim \exp (-\lambda k)$, em que $\lambda$ é a inclinação da reta semilogarítmica da distribuição de probabilidade do grau do nó. Com o valor de $\lambda$, pode-se classificar a série que origina o grafo da seguinte forma: $\lambda<\ln (3 / 2)$ está associado a um processo caótico; $\lambda=\ln (3 / 2)$ corresponde ao caso de sistemas não correlacionados; e $\lambda>\ln (3 / 2)$ caracteriza um processo estocástico correlacionado. Quanto mais acima deste limiar o Coeficiente $\lambda$ estiver, maior correlação possuirá a rede a ele associada.

\subsubsection{Coeficiente de Agrupamento}

O Coeficiente de Agrupamento $C$ de uma rede mede a probabilidade de seus nós criarem grupos unidos com uma densidade relativamente alta de conexões (Sivakumar \& Woldemeskel, 2015). Ele é definido da seguinte forma: supondo que um nó $i$ da rede tenha $k_{i}$ vizinhos que fazem parte de um mesmo grupo; isto é, que se encontram também conectados entre si, o número máximo de arestas que podem existir entre esses vizinhos é $k_{i}\left(k_{i}-1\right) / 2$. A razão entre o número $E_{i}$ de conexões que realmente existem entre esses $k_{i}$ nós vizinhos de $i$ e o número máximo de conexões possíveis $k_{i}\left(k_{i}-1\right) / 2$ fornece o Coeficiente de Agrupamento local. Isto é:

$$
C_{i}=\frac{2 E_{i}}{k_{i}\left(k_{i}-1\right)}
$$

em que, por definição, $0 \leq C_{i} \leq 1$. Além de informações sobre o agrupamento de nós, esse coeficiente permite identificar nós dominantes em uma rede complexa.

O procedimento é realizado para cada nó da rede e o valor médio do Coeficiente de Agrupamento $C$ de todos os nós é chamado de coeficiente de agrupamento médio da rede, definido por:

$$
C=\frac{1}{N} \sum_{i=1}^{N} C_{i},
$$

em que $N$ é o número total de nós da rede e $0 \leq C \leq 1$. Valores muito baixos de $C$ indicam uma rede desconectada e, portanto, aleatória, enquanto que $C=1$ indica uma rede completamente conectada (ou ordenada). Logo, esse coeficiente também pode ser usado para obter informações sobre o tipo de rede gerada.

\subsubsection{Comprimento Médio do Menor Caminho}

Uma medida de separação típica entre dois nós de um grafo é dada pelo Comprimento Médio do Menor Caminho, também conhecido como comprimento do caminho característico (Boccaletti et al., 2006). Essa medida indica o quão bem integrado é um grafo e a facilidade de se transportarem informações ou outras entidades na rede. O Comprimento Médio do Menor Caminho é definido como a média do caminho mais curto entre todos os possíveis pares de nós: $\left\langle d_{i j}\right\rangle=\frac{1}{N(N-1)} \sum_{i j} d_{i j}$, 
em que $d_{i j}$ é a menor distância entre os vértices $i$ e $j$; ou seja, o número de arestas percorridas do menor caminho para ir de $i$ até $j$ (Stam \& Reijneveld, 2007). Quanto menor for esse comprimento, mais integrada é a rede que o gerou e mais eficiente será o transporte de informação dentro dela.

\section{Metodologia}

O estudo da dinâmica de preços das commodities brasileiras soja, milho e carne de frango aqui realizado foi desenvolvido de acordo com o seguinte procedimento: com os dados que compõem as séries originais de preços dessas commodities, são geradas também as séries de retorno e de volatilidade. Aplica-se então o método Grafo de Visibilidade Horizontal (HVG) para transformar todas essas séries temporais em redes complexas, das quais são extraídos alguns índices topológicos; isto é, o Coeficiente $\lambda$ da distribuição de grau do nó, o Coeficiente de Agrupamento e o Comprimento Médio do Menor Caminho. Com essas medidas topológicas, obtêm-se informações úteis sobre a estrutura das redes geradas e se pode classificar o tipo de sistema que governa as séries temporais em estudo. Ou seja, o valor do Coeficiente de Agrupamento traz informações sobre a capacidade de agrupamento das redes, indicando seu nível de conectividade. O Comprimento Médio do Menor Caminho, por sua vez, indica o quão bem integradas são as redes; quanto menor for esse comprimento, mais bem integrada é a rede e, portanto, mais eficiente será ela na transferência de informação. E, de acordo com o valor obtido para o Coeficiente $\lambda$, é possível identificar se as séries temporais são governadas por processos caóticos $(\lambda<\ln (3 / 2))$, não correlacionados $(\lambda=\ln (3 / 2))$ ou estocásticos correlacionados $(\lambda>\ln (3 / 2))$. O algoritmo HVG foi implementado em software próprio utilizando linguagem $\mathrm{C}$ de programação.

\subsection{Descrição dos dados}

Foram utilizados os preços diários (em Reais) das commodities agrícolas brasileiras: soja, com valor em reais por saca de $60 \mathrm{~kg}(\mathrm{R} \$ / \mathrm{saca})$; milho, com valor em reais por saca de $60 \mathrm{~kg}$ ( $\mathrm{R} \$ / \mathrm{saca})$; e carne de frango, com valor em reais por quilograma $(\mathrm{R} \$ / \mathrm{kg}$ ). Os dados foram registrados no período de 03/01/2011 a 04/12/2019 (resultando em 2121 pontos para cada série) e obtidos do Centro de Estudos Avançados em Economia Aplicada - CEPEA / Escola de Agricultura Luiz de Queiroz ESALQ / Universidade de São Paulo - USP (CEPEA/ESALQ/USP, 2020). As séries temporais originais dos preços da soja, do milho e da carne de frango são mostradas na Figura 2.

Figura 2. Séries temporais originais dos preços da soja, do milho e da carne de frango.
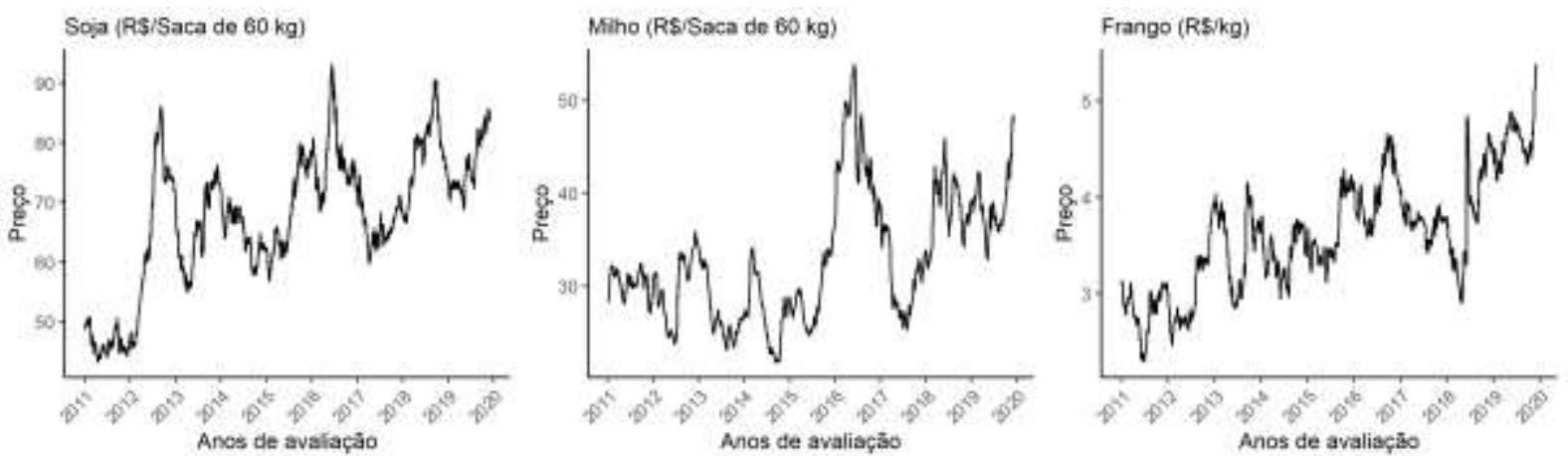

Fonte: Autores, a partir dos dados do CEPEA (2020).

Diversos estudos de séries financeiras se concentram na análise de séries de retorno (ou retorno logarítmico) por raramente apresentarem tendências ou sazonalidades (Morettin \& Toloi, 2006). A partir delas, pode-se ainda obter as séries de 
volatilidade de preços, que representam a medida de dispersão dos retornos. Define-se retorno logarítmico diário de uma série temporal como a relação $R_{t}=\ln \left(P_{t}\right)-\ln \left(P_{t-1}\right)$, onde $P_{t}$ é o preço no dia $t$. A volatilidade, por sua vez, é dada por $\left|R_{t}\right|$.

Portanto, para investigar de forma mais ampla o comportamento dinâmico dos preços das commodities aqui analisadas, foram também obtidas suas séries temporais de retorno e de volatilidade, as quais são apresentadas nas Figuras 3 e 4 , respectivamente.

Figura 3. Séries temporais de retorno dos preços da soja, do milho e da carne de frango.
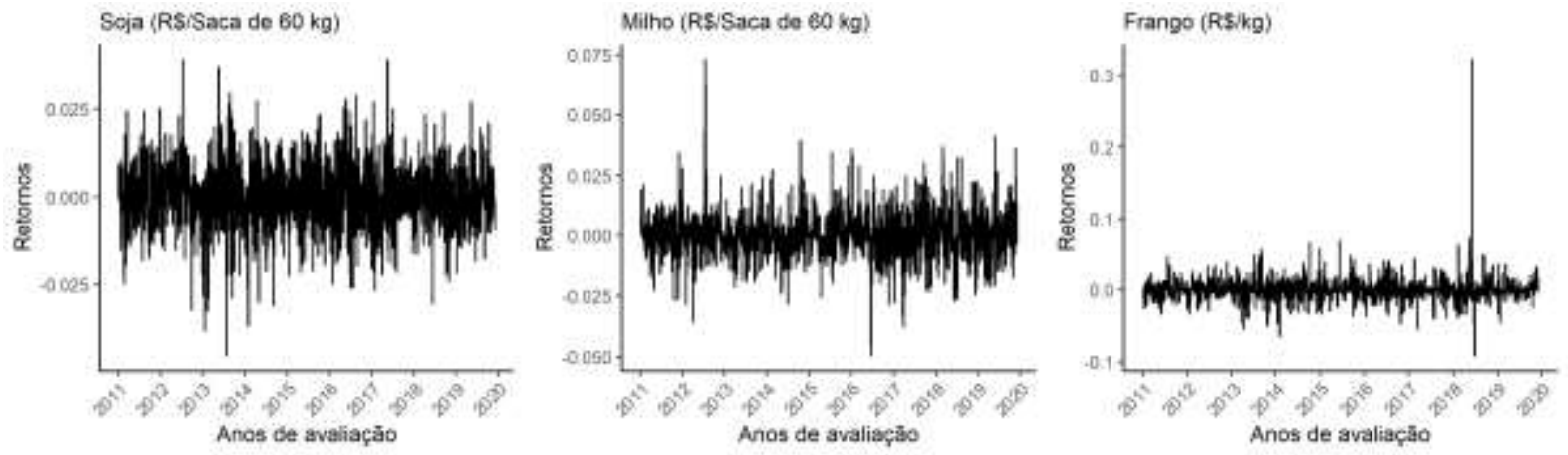

Fonte: Autores (2020).

Figura 4. Séries temporais de volatilidade dos preços da soja, do milho e da carne de frango.
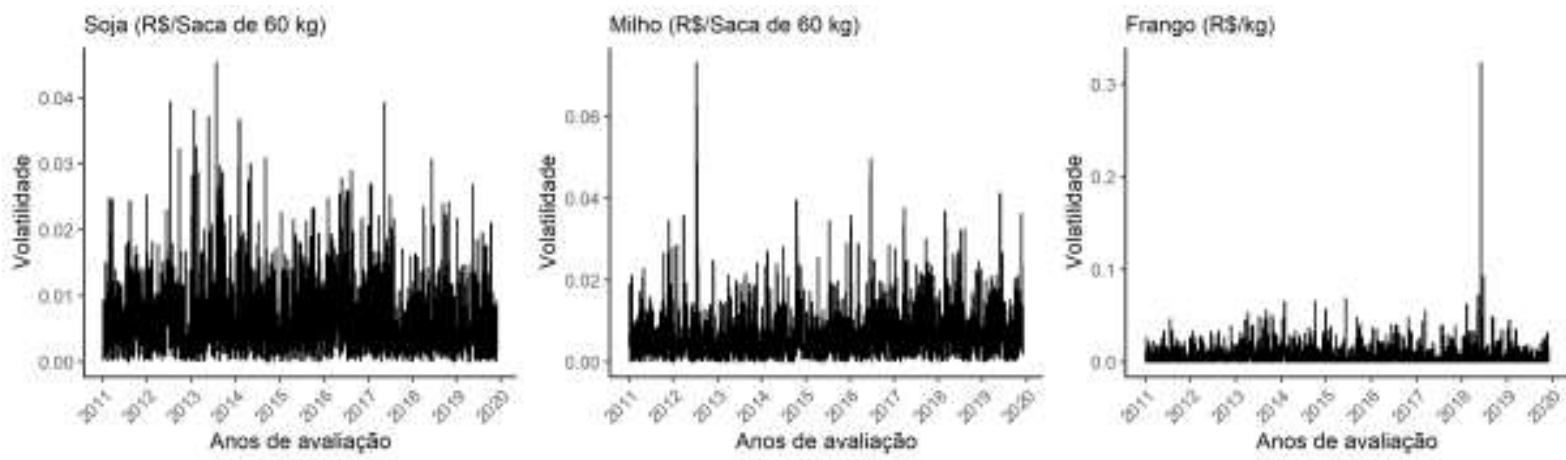

Fonte: Autores (2020).

\section{Resultados e Discussão}

Os resultados dos cálculos dos índices topológicos obtidos para as séries temporais originais de preços são apresentados na Tabela 1. Dela, vê-se que os Coeficientes de Agrupamento das redes geradas pelos grafos de visibilidade horizontal apresentaram valores próximos, exceto para a rede de frango, que apresentou um valor inferior, indicando menor nível de conectividade entre os nós do grafo. 
Tabela 1. Medidas topológicas das redes do HVG obtidas das séries temporais originais de preços da soja, do milho e da carne de frango.

\begin{tabular}{lrrr}
\hline Índices topológicos & Soja & Milho & Frango \\
\hline Coeficiente de Agrupamento & 0,5678 & 0,5495 & 0,2117 \\
Comprimento Médio do Menor Caminho & 58,4614 & 67,2644 & 40,6443 \\
Coeficiente $\lambda$ & 0,6250 & 0,5885 & 0,1790 \\
\hline
\end{tabular}

Fonte: Autores.

O Comprimento Médio do Menor Caminho indicou que as redes geradas das séries de preços da soja e do milho são menos integradas que a de frango. O Coeficiente de Agrupamento, por sua vez, mostrou que a rede desta última é menos conectada que a das duas primeiras. Além disso, as redes de preços de soja e de milho apresentaram Coeficientes de Agrupamentos próximos o bastante para indicar que elas possuem quase a mesma capacidade de agrupamento. Esta análise mostra que as redes de preço da soja e do milho apresentam características muito semelhantes, e seus resultados estão em concordância com os de Santana et al. (2020), em que os preços da soja e do milho se mostraram mais sincronizados entre si do que entre algum deles e os da carne de frango.

O valor do Coeficiente $\lambda$ (inclinação da reta semilogarítmica da distribuição de probabilidade do grau do nó) indica que as séries originais de preços da soja e do milho exibem comportamento de processos estocásticos correlacionados $(\lambda>\ln (3 / 2))$, sendo a primeira a mais correlacionada. A série original de preços da carne de frango, por sua vez, apresenta comportamento caótico $(\lambda<\ln (3 / 2))$, indicando que essa commodity apresenta maior eficiência de mercado (menor previsibilidade de preços).

Os resultados obtidos para as medidas topológicas das redes geradas pelos retornos logarítmicos são mostrados na Tabela 2. O Coeficiente de Agrupamento das redes das séries de retorno dos preços de soja e de milho apresentaram valores próximos, indicando que elas exibem capacidade de agrupamento semelhante. A rede dos preços de frango, entretanto, apresentou o menor valor para esse coeficiente, o que indica que ela é a menos conectada de todas.

Tabela 2. Medidas topológicas das redes do HVG obtidas das séries de retorno dos preços da soja, do milho e da carne de frango.

\begin{tabular}{lrrr}
\hline Índices topológicos & Soja & Milho & Frango \\
\hline Coeficiente de Agrupamento & 0,6435 & 0,6445 & 0,5001 \\
Comprimento Médio do Menor Caminho & 9,3039 & 10,3580 & 11,1134 \\
Coeficiente $\lambda$ & 0,4023 & 0,3883 & 0,4272 \\
\hline
\end{tabular}

Fonte: Autores.

Observando-se os valores obtidos para o Comprimento Médio do Menor Caminho, vê-se que a rede de retorno logarítmico dos preços diários da carne de frango foi a menos integrada, uma vez que apresentou o maior valor para esse índice topológico. Quanto ao Coeficiente $\lambda$, os valores obtidos indicam que o comportamento da série de retorno dos preços da soja tende ao de um processo não correlacionado ( $\lambda$ mais próximo do resultado para séries não correlacionadas: $\ln (3 / 2)$ ). Os retornos dos preços do milho, porém, exibiram comportamento caótico $(\lambda<\ln (3 / 2))$ e do frango, de séries estocásticas correlacionadas $(\lambda>\ln (3 / 2))$. Portanto, os retornos de preços do milho são menos previsíveis que os dos preços da soja e do frango, nessa ordem. 
As redes das séries de volatilidade, que representam a medida de dispersão dos retornos, têm suas medidas de índices topológicos apresentados na Tabela 3.

Tabela 3. Medidas topológicas das redes do HVG obtidas das séries de volatilidade dos preços da soja, do milho e da carne de frango.

\begin{tabular}{lrrr}
\hline Índices topológicos & Soja & Milho & Frango \\
\hline Coeficiente de Agrupamento & 0,6419 & 0,6336 & 0,3888 \\
Comprimento Médio do Menor Caminho & 8,6447 & 10,4507 & 11,3365 \\
Coeficiente $\lambda$ & 0,3831 & 0,4228 & 0,3911 \\
\hline
\end{tabular}

Fonte: Autores.

Os resultados mostram que esses grafos de visibilidade possuem valores próximos para o Coeficiente de Agrupamento, exceto para a série de volatilidade dos preços da carne de frango, indicando que seu grafo se mostrou menos conectado. Além disso, o grafo gerado pelo algoritmo HVG para a série de volatilidade dos preços da carne de frango mostrouse menos integrado que para o das demais séries, conforme indica o Comprimento Médio do Menor Caminho. O Coeficiente $\lambda$, por sua vez, indica que as redes de volatilidade de preços da soja e do frango apresentam características de processos caóticos $(\lambda<\ln (3 / 2))$ e, para as de preços do milho, de séries estocásticas correlacionadas $(\lambda>\ln (3 / 2))$. A variabilidade de preços da soja e da carne de frango mostram-se, portanto, menos previsíveis que a dos preços do milho.

Entre os três índices de HVG, o Coeficiente $\lambda$ é o mais útil para a escolha do modelo mais apropriado, pois é o índice com que se extraem informações sobre a natureza dos processos que governam as séries temporais em análise. Os modelos que geram séries caóticas são os mais adequados para estudar a dinâmica de preços da carne de frango, do retorno de preços do milho, e de volatilidade de preços da soja e da carne de frango; e modelos que geram séries correlacionadas para preços de soja e de milho, retorno de preços da carne de frango e volatilidade de preços do milho. A Tabela 4 traz um resumo da classificação apresentada.

Tabela 4. Classificação das séries temporais de preço, retorno e volatilidade das commodities agrícolas brasileiras soja, milho e carne de frango.

\begin{tabular}{llll}
\hline Tipo de série & Soja & Milho & Carne de frango \\
\hline Caótica $(\lambda<\ln (3 / 2))$ & Série de volatilidade & Série de retorno & $\begin{array}{l}\text { Série de preços e de } \\
\text { volatilidade }\end{array}$ \\
Estocástica correlacionada $(\lambda>\ln (3 / 2))$ & Série de preços & $\begin{array}{l}\text { Série de preços e de } \\
\text { volatilidade }\end{array}$ & Série de retorno \\
\hline
\end{tabular}

Fonte: Autores.

\section{Considerações Finais}

A análise dos índices topológicos das redes geradas pelo HVG mostrou que a série de preços originais da carne de frango apresenta uma rede mais integrada que as redes dos insumos soja e milho, conforme resultados obtidos para o Comprimento Médio do Menor Caminho. As duas últimas redes, entretanto, apresentaram maior capacidade de agrupamento, característica de redes mais conectadas, conforme mostram seus coeficientes de agrupamento. Também as redes de retorno e volatilidade de preços desses insumos se mostraram mais conectadas. Esses resultados indicam que os grafos de visibilidade horizontal da soja e do milho possuem propriedades estruturais muito semelhantes. Quanto ao Coeficiente $\lambda$, seus valores indicaram que as séries temporais dos preços da soja e do milho são geradas por processos estocásticos correlacionados. Isso 
significa que, entre o ano de 2011 e final de 2019, os preços dessas commodities eram mais previsíveis, indicando que, durante esse período, os mercados brasileiros da soja e do milho se mostravam menos eficientes, ao contrário do que acontecia com o mercado da carne de frango, já que a série de preços dessa commodity traduz um processo caótico, indicando preços menos previsíveis. As séries de retorno dos preços da carne de frango e a série de volatilidade dos preços do milho são também geradas por processos estocásticos correlacionados. A série de retorno de preços da soja, por sua vez, apresenta comportamento que tende ao de um processo não correlacionado, e as séries de volatilidade de preços da soja e da carne de frango são geradas por processos caóticos. A natureza das séries de volatilidade dessas duas últimas commodities indica, portanto, uma variabilidade de preços menos previsível.

Os resultados acima descritos podem ser úteis para o desenvolvimento e validação de modelos teóricos e computacionais para a previsão de preços das commodities agrícolas brasileiras soja, milho e carne de frango. O método Grafo de Visibilidade Horizontal, até então não aplicado ao estudo da dinâmica de preços de produtos agrícolas, mostrou-se, portanto, eficiente quanto à captura de propriedades estruturais das redes geradas e à classificação das séries em relação ao tipo de processo que as governa.

Em trabalhos futuros, pode-se explorar outras medidas topológicas dos grafos de visibilidade ou utilizá-los para estudar o comportamento de outras commodities agrícolas brasileiras, como também aplicar variações do método Grafo de Visibilidade que estão sendo amplamente discutidas na literatura.

\section{Agradecimentos}

Os autores agradecem às seguintes instituições pelo apoio prestado para que esta pesquisa fosse possível: Universidade Federal Rural de Pernambuco (UFRPE); Programa de Pós-Graduação em Biometria e Estatística Aplicada (PPGBEA); Coordenação de Aperfeiçoamento de Pessoal de Nível Superior (CAPES); Conselho Nacional de Desenvolvimento Científico e Tecnológico (CNPq); Fundação de Amparo à Ciência e Tecnologia do Estado de Pernambuco (FACEPE); e Centro de Estudos Avançados em Economia Aplicada (CEPEA).

\section{Referências}

Ahmadi, N., \& Pechenizkiy, M. (2016). Application of horizontal visibility graph as a robust measure of neurophysiological signals synchrony. Proceedings IEEE Symposium on Computer-Based Medical Systems, 2016-August, 273-278. https://doi.org/10.1109/CBMS.2016.73

Albert, R., \& Barabási, A.-L. (2002). Statistical mechanics of complex networks. Reviews of Modern Physics, 74(1), 47-97. https://doi.org/10.1103/RevModPhys.74.47

Bini, D. A., Canever, M. D., De Souza, M. O., \& Ely, R. A. (2016). Transmissão De Preços Ao Longo Das Cadeias Produtivas Do Brasil. Revista de Economia, 42(1). https://doi.org/10.5380/re.v42i1.48660

Boccaletti, S., Latora, V., Moreno, Y., Chavez, M., \& Hwang, D. U. (2006). Complex networks: Structure and dynamics. Physics Reports, 424(4-5), 175-308. https://doi.org/10.1016/j.physrep.2005.10.009

Braga, A. C., Alves, L. G. A., Costa, L. S., Ribeiro, A. A., De Jesus, M. M. A., Tateishi, A. A., \& Ribeiro, H. V. (2016). Characterization of river flow fluctuations via horizontal visibility graphs. Physica A: Statistical Mechanics and Its Applications, 444, 1003-1011. https://doi.org/10.1016/j.physa.2015.10.102

CEPEA/ESALQ/USP. (2020). Consultas ao Banco de Dados do Site - Centro de Estudos Avançados em Economia Aplicada - CEPEA-Esalq/USP. Recuperado de https://www.cepea.esalq.usp.br/br/consultas-ao-banco-de-dados-do-site.aspx

CNA. (2020). Panorama do Agro | Confederação da Agricultura e Pecuária do Brasil (CNA). https://www.cnabrasil.org.br/cna/panorama-do-agro\#_ftn2

EMBRAPA. (2020). Desempenho de produção 2019. EMBRAPA - Suínos e Aves. https://www.embrapa.br/suinos-e-aves/cias/estatisticas

FAS/USDA (2020). United States Department of Agriculture Foreign Agricultural https://apps.fas.usda.gov/psdonline/app/index.html\#/app/topCountriesByCommodity\#chart28. Acessado em 27 de agosto de 2020.

Gao, Z. K., Cai, Q., Yang, Y. X., Dang, W. D., \& Zhang, S. S. (2016). Multiscale limited penetrable horizontal visibility graph for analyzing nonlinear time series. Scientific Reports, 6(October), 1-7. https://doi.org/10.1038/srep35622 
Gouveia, A. B. V. S., de Paulo, L. M., da Silva, J. M. S., de Sousa, F. E., dos Santos, F. R., Minafra, C. S. (2020). Subprodutos da soja na alimentação de aves: Revisão. Research, Society and Development, 9(7), e471974187. http://dx.doi.org/10.33448/rsd-v9i7.4187

Hochman, G., Rajagopal, D., Timilsina, G., \& Zilberman, D. (2014). Quantifying the causes of the global food commodity price crisis. Biomass and Bioenergy, 68, 106-114. https://doi.org/10.1016/j.biombioe.2014.06.012

Ivanic, M., Martin, W., \& Zaman, H. (2012). Estimating the Short-Run Poverty Impacts of the 2010-11 Surge in Food Prices. World Development, 40(11), 2302-2317. https://doi.org/10.1016/j.worlddev.2012.03.024

Lacasa, L., Luque, B., Ballesteros, F., Luque, J., \& Nuno, J. C. (2008). From time series to complex networks: The visibility graph. Proceedings of the National Academy of Sciences, 105(13), 4972-4975.

Lacasa, L., \& Toral, R. (2010). Description of stochastic and chaotic series using visibility graphs. Physical Review E - Statistical, Nonlinear, and Soft Matter Physics, 82(3), 1-11. https://doi.org/10.1103/PhysRevE.82.036120

Lange, H., Sippel, S., \& Rosso, O. A. (2018). Nonlinear dynamics of river runoff elucidated by horizontal visibility graphs. Chaos, 28(7), 075520. https://doi.org/10.1063/1.5026491

Luque, B., Lacasa, L., Ballesteros, F., \& Luque, J. (2009). Horizontal visibility graphs: Exact results for random time series. Physical Review E - Statistical, Nonlinear, and Soft Matter Physics, 80(4), 1-11. https://doi.org/10.1103/PhysRevE.80.046103

Madl, T. (2016). Network analysis of heart beat intervals using horizontal visibility graphs. Computing in Cardiology, 43, 733-736. https://doi.org/10.22489/cinc.2016.213-510

Morettin, P., \& Toloi, C. (2006). Análise de Séries Temporais. Egard Blucher.

Oliveira Junior, O. D. P., Wander, A. E., \& Figueiredo, R. S. (2014). Relação entre os preços do milho, da soja e da carne de frango no período de 2004 a 2013. In Embrapa Arroz e Feijão-Artigo em anais de congresso (ALICE). In: Congresso Da Sociedade Brasileira De Economia, Administração E Sociologia Rural, 52, 2014, Goiânia. Heterogeneidade e suas implicações no rural brasileiro: anais. Goiânia: Sober, 2014.

Santana, L. I. T. de, da Silva, J. M., Araújo, L. S., Moreira, G. R., \& Stosic, T. (2020). Análise de quantificação de recorrência de preços brasileiros do milho, da soja e da carne de frango. Research, Society and Development, 9(10), e9979109461. https://doi.org/10.33448/rsd-v9i10.9461

Sivakumar, B., \& Woldemeskel, F. M. (2015). A network-based analysis of spatial rainfall connections. Environmental Modelling and Software, 69, 55-62. https://doi.org/10.1016/j.envsoft.2015.02.020

Stam, C. J., \& Reijneveld, J. C. (2007). Graph theoretical analysis of complex networks in the brain. In Nonlinear Biomedical Physics. 1(1), 1-19. BioMed Central. https://doi.org/10.1186/1753-4631-1-3

Vamvakaris, M. D., Pantelous, A. A., \& Zuev, K. M. (2018). Time series analysis of S\&P 500 index: A horizontal visibility graph approach. Physica A: Statistical Mechanics and Its Applications, 497, 41-51. https://doi.org/10.1016/j.physa.2018.01.010

Zhang, J., Sun, J., Luo, X., Zhang, K., Nakamura, T., \& Small, M. (2008). Characterizing pseudoperiodic time series through the complex network approach. Physica D: Nonlinear Phenomena, 237(22), 2856-2865. https://doi.org/10.1016/j.physd.2008.05.008

Zhu, G., Li, Y., \& Wen, P. P. (2014). Analysis and classification of sleep stages based on difference visibility graphs from a single-channel EEG signal. IEEE Journal of Biomedical and Health Informatics, 18(6), 1813-1821. https://doi.org/10.1109/JBHI.2014.2303991

Zou, Y., Donner, R. V., Marwan, N., Donges, J. F., \& Kurths, J. (2019). Complex network approaches to nonlinear time series analysis. Physics Reports, 787, 1-97. https://doi.org/10.1016/j.physrep.2018.10.005 